\title{
Determinação da diversidade fenotípica e genotípica de Salmonella enterica subsp. enterica SOROVAR Enteritidis no Rio Grande do Sul*
}

\author{
CLARISSA SILVEIRA LUIZ VAZ
}

Cláudio Wageck Canal (Orientador - UFRGS)
Marisa Ribeiro de Itapema Cardoso (Co-Orientadora - UFRGS)

Banca: Angelo Berchieri Junior (Unesp), Antonio José Piantino Ferreira (USP), Gertrudes Corção (UFRGS), Eduardo César Tondo (UFRGS)

Salmonella enterica sorovar (S.) Enteritidis é uma das principais bactérias envolvidas em surtos de infecção alimentar em humanos decorrentes do consumo de produtos de origem animal. Nas investigações epidemiológicas, a subtipificação fenotípica e genotípica permitem verificar diferenças ou similaridades entre linhagens, as quais podem indicar sua origem, auxiliando os programas de controle e prevenção do patógeno. No presente trabalho, foram analisados os padrões de resistência a antimicrobianos, fagotipificação, macrorestrição de DNA seguida de eletroforese em campo pulsado (PFGE) e polimorfismo de comprimento do fragmento amplificado utilizando uma única enzima de restrição (SE-AFLP) de 107 linhagens de $S$. Enteritidis, sendo 53 isoladas de aves e seus produtos (carcaças, carne, vísceras e ambiente) e uma de origem suína. Foram ainda analisadas 29 linhagens isoladas de alimentos e 14 isoladas de humanos envolvidos em surtos de salmonelose notificados no Rio Grande do Sul, uma amostra de referência (ATCC 13076), além de 9 linhagens isoladas na Europa e África. Na comparação entre amostras isoladas de aves e de surtos de salmonelose, as linhagens de origem avícola foram significativamente mais resistentes a antimicrobianos do que as isoladas de humanos e, embora não tenha sido encontrada relação entre os genótipos de PFGE e os padrões de resistência, a associação de ambas as técnicas possibilitou uma melhor caracterização de $S$. Enteritidis, podendo ser úteis como marcadores epidemiológicos. Por outro lado, SE-AFLP agrupou a maioria das linhagens de $S$. Enteritidis num padrão principal. Paralelamente, na comparação de técnicas genotípicas com base em PCR, SE-AFLP apresentou maior capacidade de discriminação, embora inferior à verificada pela fagotipificação e perfil de resistência a antimicrobianos. Assim, foi recomendado que esta técnica deve ser utilizada em associação com outras metodologias na tipificação de $S$. Enteritidis. Posteriormente, na caracterização do conjunto de linhagens de $S$. Enteritidis, foram identificados 13 diferentes padrões de resistência a antimicrobianos, além de 12 padrões de PFGE e 3 de SE-AFLP. As técnicas genotípicas demonstraram que existe um genótipo predominante de $S$. Enteritidis no Rio Grande do Sul, o qual está presente em aves e produtos relacionados, além de alimentos e humanos envolvidos em surtos de salmonelose, sugerindo que estas linhagens possam derivar de uma fonte comum, tendo provável relação clonal. Embora havendo um genótipo predominante de $S$. Enteritidis, a associação das técnicas propostas possibilitou a melhor caracterização do patógeno.

Descritores: Salmonella Enteritidis, genotipificação, antimicrobianos, fagotipo, epidemiologia.

*Tese de Doutorado n. 81 (Especialidade: Bacteriologia). 136f. Programa de Pós-graduação em Ciências Veterinária [www.ufrgs.br/ppgcv], Faculdade de Veterinária, Universidade Federal do Rio Grande do Sul (UFRGS), Porto Alegre, RS/Brasil. CORRESPONDÊNCIA: C.W. Canal [claudio.canal@ufrgs.br]. 


\title{
Phenotypic and genotypic diversity of Salmonella enterica subsp.enterica SOROVAR Enteritidis isolated in Rio Grande do Sul, Brazil**
}

\author{
CLARISSA SILVEIRA LUIZ VAZ
}

\author{
Cláudio Wageck Canal (Adviser - UFRGS)
}

Marisa Ribeiro de Itapema Cardoso (Co-Adviser - UFRGS)

Committee: Angelo Berchieri Junior (Unesp), Antonio José Piantino Ferreira (USP), Gertrudes Corção (UFRGS), Eduardo César Tondo (UFRGS)

Salmonella enterica subsp. enterica (S.) serovar Enteritidis is one of the main pathogens involved in food-borne diseases worldwide. Subtyping is crucial to differentiate strains, as well to study its origin and major infection route, which might direct preventive and control measures in epidemiological investigations of food-related salmonellosis. In order to investigate phenotypic and genotypic characterization in S. Enteritidis, the aim of this study was analyze the antimicrobial resistance, phage types, pulsed-field gel elecrophoresis (PFGE) and single-enzyme amplified-fragment length polymorphism (SE-AFLP) patterns of $107 \mathrm{~S}$. Enteritidis strains isolated from poultry (53 isolates from carcass, meat, viscera and environmental swab) and one from swine. We also analyze 29 strains from food and 14 from humans involved in salmonellosis outbreaks obtained in Rio Grande do Sul, one reference strain (ATCC 13076) and 9 strains isolated from African and European countries. Resistance rates found in poultry-related strains were significantly higher than that observed in strains isolated from humans. Since identical PFGE profiles were identified in antimicrobial resistant and susceptible strains, it was not possible to find relationship between the PFGE genotypes and the antimicrobial resistance patterns found. However, both approaches were able to improve S. Enteritidis characterization. On the other hand, SE-AFLP clustered most of S. Enteritidis strains analyzed in a major pattern. In a comparison between based-PCR approaches, SE-AFLP showed a higher discriminatory power, although it was lower than that displayed by antimicrobial resistance and phage type patterns. Thus, SE-AFLP might be used associated to other approaches to S. Enteritidis characterization. In contrast of, 13 different antimicrobial resistance patterns, 12 PFGE genotypes and 3 SE-AFLP genotypes were identified in S. Enteritidis strains. Genotypic approaches showed that strains isolated from poultry, poultry-related products, food and humans involved in salmonellosis outbreaks shared a major pattern. These results suggest that should be a close relationship between contamination sources and these associated approaches improved S. Enteritidis characterization.

Key words: Salmonella Enteritidis, genotyping, antimicrobial, phage type, epidemiology.

Presented: 28 February 2007

\footnotetext{
**Doctoral Dissertation \# 81 (Field: Bacteriology) 136p. Graduate Program in Veterinary Sciences [www.ufrgs.br/ppgcv], Faculdade de Veterinária, Universidade Federal do Rio Grande do Sul (UFRGS), Porto Alegre, RS/Brazil. CORRESPONDENCE: C.W. Canal [claudio.canal@ufrgs.br].
} 